\title{
Immunological and Inflammatory Indicators of COVID-19 Patients With Returned-positive Nucleic Acid Tests During Hospitalization: a Retrospective Cohort Study
}

\section{Yin Wang}

Children's Hospital of Fudan University

Xiaobei Chen

Renmin Hospital of Wuhan University

Yi Zhang

Children's Hospital of Fudan University

Hongyan Chen

Children's hospital of fudan university

Quan Zhou

Renmin Hospital of Wuhan University

Dong Li

Renmin Hospital of Wuhan University

Zhili Niu

Renmin Hospital of Wuhan University

Huidan Yu

Wuhan University

Xiaojun Wang

Hubei Provincial Women's and Children's Hospital

Haijun Zhang

Hubei Provinical Women's and Children's Hospital

Tingting Liu

Hubei Provincial Women's and Children's Hospital

Bicheng Zhang

Renmin Hospital of Wuhan University

Hui Yu

Children's Hospital of Fudan University

Xiaochuan Wang

Children's Hospital of Fudan University

\section{Yuan Jiang}

Children's Hospital of Fudan University 


\section{Yalan Dou}

Children's Hospital of Fudan University

Xiaotian Chen

Children's Hospital of Fudan University

Xiaoyang Zhou

Renmin Hospital of Wuhan University

Weili Yan ( $\square$ yanwl@fudan.edu.cn )

Children hospital of Fudan University

\section{Research article}

Keywords: COVID-19, SARS-CoV-2, returned-positive, the third component of complement, procalcitonin, anti-SARS-CoV-2 IgM, anti-SARS-CoV-2 IgG

Posted Date: January 13th, 2021

DOl: https://doi.org/10.21203/rs.3.rs-142839/v1

License: (a) (i) This work is licensed under a Creative Commons Attribution 4.0 International License. Read Full License 


\section{Abstract}

Background: COVID-19 cases with suspected returned-positive SRAS-CoV-2 tests following consecutive negative tests have been reported, but evidence-based explanations for this phenomenon is still lacking. We aimed to describe the clinical and laboratory characteristics of returned-positive COVID-19 patients during treatment in comparison with other patients.

Methods: From January 20 to April 10, 2020, all COVID-19 inpatient with at least three RT-PCR SARS-CoV2 tests in Renmin Hospital in Wuhan, China were enrolled. Patients with 2 consecutively negative RT-PCR results followed by a positive result were classified as returned-positive patients, and their characteristics and repeatedly measured laboratory results were compared with the rest of the patients. Linear mixed effects models were performed.

Results: A total of 789 COVID-19 patients were included and 22.8\% patients returned positive in RT-PCR SARS-CoV-2 test. No significant differences were found for general characteristics between the returnedpositive and the control groups. The trends of inflammatory and immune factors including the third component of complement (C3), C-reactive protein, procalcitonin (PCT), IL-4, IL-6, the counts of lymphocyte, $\mathrm{CD} 3+, \mathrm{CD} 8+$, white blood cell and immunoglobulin levels during hospitalization were significantly different between the two groups. During the returned-positive period, C3, PCT, serum IgM, anti-SARS-CoV-2 IgM and anti-SARS-CoV-2 IgG were significantly higher in the returned-positive patients at certain time points.

Conclusions: Returned-positive COVID-19 patients appeared to be more sever at admission, and had periodically higher levels in C3, PCT, serum IgM and two specific antibodies during hospitalization. This suggests that positive return of SARS-COV-2 could not be completely explained by false-negative testing and longer observation of these patients is warranted.

\section{Background}

A pandemic of coronavirus disease (COVID-19) has threatened public health globally since December 2019, which is caused by severe acute respiratory syndrome coronavirus 2 (SARS-CoV-2). The pandemic of COVID-19 has threatened public health globally with more than 18 million infected cases and new cases rising by around 250000 each day, according to World Health Organization (WHO), by August 4, 2020 [1]. As a novel disease, no specific treatment has been validated for COVID-19 so far, and traditional public health tactics including isolation, quarantine and community containment are critical to controlling the spread of the disease $[2,3]$. WHO also continues to urge countries to increase testing and contact tracing to ensure no cases are missed [1].

Current guideline suggests two consecutively negative real-time reverse transcriptase polymerase chain reaction (RT-PCR) results of SARS-CoV-2 testing as one of the criteria for discharge [4]. However, several reports show that a small portion of such recovered patients tested positive for infection through the nucleic acid test again during a follow-up visit. Experts tend to consider it as the failure of SARS-CoV-2 
testing, including failure during sampling or false negative RT-PCR test [5]. Recently, Lan and colleague have reported the presence of positive RT-PCR test in four recovered COVID-19 patients [6], arguing against the possibility of the false negative SARS-CoV-2 testing. However, evidence-based explanations based on a large sample of patient data on this phenomenon are still lacking.

Thus, we utilized data of 789 COVID-19 inpatients from Renmin Hospital located in Wuhan, China, from January 20 to April 10, 2020, to describe immunological and inflammatory indicators of patients with returned-positive SRAS-CoV-2 tests compared with their counterparts.

\section{Methods}

\section{Patients}

From January 20 to April 10, 2020, all COVID-19 inpatient with at least three SARS-CoV-2 laboratory results were eligible and recruited for this study from Renmin Hospital of Wuhan University, which is one of the major tertiary teaching hospitals and government-authorized hospitals for COVID-19 patients.

\section{Diagnosis of COVID-19}

All COVID-19 patients were diagnosed and clinically graded according to the interim guidance from WHO [7] and National Health Commission of China [4]. The diagnosis of COVID-19 was made based on a positive result of nasopharyngeal swab by respiratory pathogen nucleotide test with RT-PCR [8]. According to clinical symptoms, imaging findings and laboratory results, COVID-19 patients were classified into four types: mild, moderate, severe and critical [4].

\section{Data collection}

Clinical data of patients were obtained from the hospital electronic medical record system, including demographics, severity of COVID-19, comorbidities (including respiratory diseases, cardiovascular diseases, cerebrovascular diseases, kidney and urinary diseases, digestive system diseases, liver and gallbladder diseases, metabolic diseases, immune diseases, nerve and mental system diseases, tumors and operation history), laboratory examinations, anti-virus treatments, prognosis and inflammatory and immunologic markers.

\section{Definitions of returned-positive patients}

Returned-positive patients were defined as patients with two consecutively negative RT-PCR results of SARS-CoV-2 tests followed by a positive result (a certain sequence of results: negative, negative, positive), with an interval of at least 24 hours between RT-PCR tests. The rest of the enrolled COVID-19 patients were included in the control group.

\section{Laboratory testing}


The COVID-19 laboratory test assays were conducted according to the WHO recommendation. RNA was extracted and tested by RT-PCR with COVID-19 specific primers and probes. The virus genome sequence was examined in samples of bronchoalveolar-lavage fluid by one of three methods: Sanger sequencing, Illumina sequencing, or nanopore sequencing.

Specific antibodies against IgG and IgM were detected using Human SARS-CoV-2 IgG and IgM Chemiluminescence Analysis (CLIA) Assays panel (Shenzhen YHLO Biotech Co., Ltd., Shenzhen, China) and the high-speed CLIA system iFlash 3000 (Shenzhen YHLO Biotech Co., Ltd., Shenzhen, China). Proinflammatory cytokines including interleukin (IL)-2, IL-4, IL-6 and IL-10 were detected using Human Cytokine Standard Assays panel (ET Healthcare, Inc., Shanghai, China) and the Bio-Plex 200 system (BioRad, Hercules, CA, USA) according to the instructions. NLR was calculated by dividing the absolute neutrophil count by the lymphocyte count.

\section{Statistical analysis}

Descriptive analyses were used to summarize the patients' epidemiological and clinical features. Continuous variables were presented as the median and interquartile range (IQR) as appropriate, and categorical variables were expressed as absolute numbers and percentages. Chi-squared tests or Fisher's exact tests were adopted for group comparison of categorical variables.

From the date of confirmed diagnosis of SARS-CoV-2 infection, the course of disease (days) was ordinally categorized into phases by an interval of 5 days. Linear mixed effect models were used to analyze the difference in trends of laboratory markers over time (by phase) between returned-positive patients and their counterparts. We entered treatment, gender, age, disease conditions and antiviral therapy (with interaction term of group and phase) as fixed effect into the model. Patients ID was intercepted as random effects. The significance of group differences in the overall trends of the biomarkers was reported and margins effects (means and 95\% confidence interval) were plotted over phases accordingly. All analyses were performed using STATA 16.0 software (Stata Corporation, College Station, TX, USA). A two-sided $p$ value less than 0.05 was considered statistically significant.

\section{Results}

From January 29 to April 1, 2020, there were 1098 inpatients with laboratory-confirmed SARS-CoV-2 in Renmin Hospital in Wuhan, China. Among them, 789 (71.9\%, 789/1098) COVID-19 patients with at least three RT-PCR tests of SARS-CoV-2 were eligible for the final analysis, of which $22.8 \%$ (180/789) patients returned positive of SARS-CoV-2 during hospitalization (Fig. 1). General characteristics of patients at admission to hospital, including age, gender and comorbidities, did not significantly differ between the patients included and those excluded. However, higher mortality and shorter length of hospitalization were observed patients not included in the study (See Supplementary Table 1, Additional File 1).

The mean (SD) age of the included patients was 59.3(SD: 13.7) years old. Among them, 389 (49.3\%) were males, and $56.6 \%$ had comorbidities. Compared with the control group, the returned-positive group had 
similar age, proportion of gender and prevalence of comorbidities, but had more severe patients at admission ( $87.2 \%$ vs. $77.2 \%, p=0.003)$. During hospitalization, the proportions of patients receiving Abidal hydrochloride treatment ( $88.9 \%$ vs. $77.9 \%)$, hydroxychloroquine treatment $(28.3 \%$ vs. $23.9 \%)$, or not receiving any anti-virus treatment $(27.2 \%$ vs. $0.7 \%)$ were significantly larger in returned-positive patients than the control groups $(p \leq 0.017)$. On average, positive return of SARS-CoV-2 occurred at median day 27 (IQR: 20-34 day) after diagnosis, and these patients stayed 8 days longer in hospital compared with their counterpart patients (Table 1).

Table 1

Characteristics of enrolled COVID-19 patients.

\begin{tabular}{|c|c|c|c|}
\hline & $\begin{array}{l}\text { Returned-positive }(\mathrm{N}= \\
\text { 180) }\end{array}$ & $\begin{array}{l}\text { Control group } \\
(\mathrm{N}=609)\end{array}$ & $\mathbf{P}$ \\
\hline Age, (year) & $60.2 \pm 13.2$ & $58.2 \pm 14.4$ & 0.25 \\
\hline Male, $n,(\%)$ & $85(47.2)$ & $304(49.8)$ & 0.54 \\
\hline Comorbidity $^{\mathrm{a}}, \mathrm{n} / \mathrm{N}(\%)$ & 101/178(56.7) & $346 / 607(57.0)$ & 0.95 \\
\hline Covid-19 Classification ,n(\%) & 180 & 609 & \\
\hline Moderate & $14(7.8)$ & $110(18.1)$ & 0.004 \\
\hline Severe & 157(87.2) & $470(77.2)$ & \\
\hline Critical & $9(5.0)$ & $29(4.7)$ & \\
\hline $\begin{array}{l}\text { Received Abidal Hydrochloride treatment, } \\
\mathrm{n}(\%)\end{array}$ & $160(88.9)$ & $475(77.9)$ & 0.002 \\
\hline $\begin{array}{l}\text { Received Hydroxychloroquine treatment, } \\
n(\%)\end{array}$ & $51(28.3)$ & $146(23.9)$ & 0.017 \\
\hline $\begin{array}{l}\text { Did not Receive any anti-virus treatments, } \\
\mathrm{n}(\%)\end{array}$ & $49(27.2)$ & $4(0.7)$ & $<0.0001$ \\
\hline \multicolumn{4}{|l|}{ Prognosis, $\mathrm{n}(\%)$} \\
\hline Death & $3(1.7)$ & $18(3.0)$ & 0.23 \\
\hline Cured and discharged & 177(98.3) & $590(93.7)$ & \\
\hline Transferred to other hospital & $0(0.0)$ & $2(0.3)$ & \\
\hline Length of Hospitalization, day & $37(31-44)$ & $29(21-38)$ & 0.009 \\
\hline $\begin{array}{l}\text { Length of SARS-CoV-2 returned-positive, } \\
\text { day }\end{array}$ & \multicolumn{3}{|l|}{$27(20-34)$} \\
\hline \multicolumn{4}{|c|}{$\begin{array}{l}\text { a: Comorbidity including respiratory diseases, cardiovascular diseases, cerebrovascular diseases, } \\
\text { kidney and urinary diseases, digestive system diseases, liver and gallbladder diseases, metabolic } \\
\text { diseases, immune diseases, nerve and mental system diseases, tumors and operation history. }\end{array}$} \\
\hline
\end{tabular}


The availability of the laboratory data varied over the overall 11 phases and among biomarkers. Statistically significant differences in the overall trends by phase of several inflammatory and immunologic markers during hospitalization were found between the two patient groups $(p<0.05)$. These included the third component of complement (C3), C-reactive protein (CRP), procalcitonin (PCT), interleukin-4 (IL-4), interleukin-6 (IL-6), the counts of lymphocyte (LYM) and white blood cell (WBC) (Fig. 2), and the counts of CD3 + and CD8+, serum antibodies of $\lg A, \lg E$, $\lg M$, and serum anti-SARS-CoV-2 IgG, anti-SARS-CoV-2 IgM (Fig. 3). While we observed one or more significant differences at certain phases during the hospitalization, we did not find any biomarker with unidirectional group differences. For instance, the returned-positive patients had significantly higher C3 components at phase $6(p=0.015)$, and higher CRP at phase $2(p=0.009)$ and phase $3(p=0.033)$ as compared with the control group. The LYM counts from phase 1 to 6 of the returned-positive group was non-significantly lower than the control group, but became significantly higher at phase $9(p=0.009)$. This group of patients also showed higher PCT at phase 7,11 and WBC counts at phase 8,10 than the controls, respectively, with no significant difference during the rest of phases (Fig. 2C, D). Although a significant overall trend of IL-4 between the two groups was indicated, no statistical differences were found for any phases. Significant higher IL-6 and IL-10 levels at phase 10 were both shown in the returned-positive group $(p<0.001)($ Fig. 2F, G, H).

The CD3 + and CD8 + counts were significantly higher in the returned-positive group from phase 1 to 3 and at phase 11 (Fig. 3A, B). An overall higher serum IgA levels was also seen in this group except for phase 2 only $(p=0.034)$ (Fig. 3C). Reversely, serum IgE were significantly lower at phase 1 only $(p=$ 0.005) (Fig. 3D).

Overall, IgM levels in the returned-positive group were significantly higher than those in the control group except for phase 2. Only at phase 2, serum IgM levels in the returned-positive were significantly lower (Fig. 3E). On the other hand, anti-SARS-CoV-2 IgM in the returned-positive group was higher than the control group, with significant differences found at phase 1, 2, 4, 5, 7 and 9 (Fig. 3G). Furthermore, antiSARS-CoV-2 IgG were higher in returned-positive group during the early and late phases but similar during the middle phases, but with significance at phase 1,9 and 10. Contrarily, it became significantly lower at the last phase (Fig. 3F). Results of other markers with non-significant results for the overall models were displayed in the appendix tables and figures. (See Supplementary Fig. 1 to Fig. 4, Additional File 1)

\section{Discussion}

This is the first report with quantitative data on more than 20 biomarkers evaluating the returned-positive phenomenon of SARS-CoV-2 in a large cohort of 789 COVID-19 patients. We found that returned-positive patients show differences in several inflammatory and immunologic markers during the disease course. Especially, compared with the control group, serum IgM, anti-SARS-CoV-2 IgM, anti-SARS-CoV-2 IgG, C3, PCT were significant higher at certain periods (median at day 27, IQR: day 20-34). 
Convalescent plasma from cured COVID-19 patients has been observed with some effect in clinical treatments [9-11]. Although the cut-off concentration of anti-SARS-CoV-2 IgG to ensure the therapeutic potency of the convalescent plasma remains unknown, serum antibody titer, which strongly correlates to antibody concentration, has been proposed to be 1:320 or above [9-11]. This may explain the increased anti-SARS-CoV-2 IgG levels found in both groups during the later course of disease (i.e., day 40 to 50); these patients might be on the process of recovering, producing neutralizing specific antibodies effectively against further infection. Interestingly, the increment of anti-SARS-CoV-2 IgG levels was greater for the return-positive group. In line with this, anti-SARS-CoV-2 IgM levels appeared to be higher in the returned-positive group consisting of more severe patients during the entire hospitalization. As shown in our further subgroup analyses, the anti-SARS-CoV-2 IgM levels were the highest in the patients who died before 45 days, followed by patients with the longest hospitalization (up to 55 days), and were lowest in the patients who were discharged before 45 days. Although counterintuitive, these findings are analogous to a recent analysis of immunological features of asymptomatic and symptomatic SARS-CoV-2 infections [12].

In that study, anti-SARS-CoV-2 IgG levels in the symptomatic group were significantly higher than those in the asymptomatic group. We postulated that the difference in intensity of the two specific antibodies between those returned positive and those did not might suggest a different immune response to SARSCoV-2 virus. However, given the relatively few patients included in this analysis, whether these phenomena are true and the underling biological mechanisms warrant further studies.

To our knowledge, there is no detailed report on whether complement would be a potential target for COVID-19 patients. Evidence from an animal experiment has demonstrated that the activation of C3 exacerbates disease in SARS-CoV-associated ARDS [13]. In addition, loss-of-function experiment showed that mice a lack of the $\mathrm{C} 3$ protein results in improved lung function, and less cytokine release [13]. Consistent with these findings, our results showed that C3 was significantly higher in returned-positive group approximately one month after infection.

The PCT production and its release into the circulation are enormously amplified during bacterial infections. In a recent meta-analysis, increased PCT values were linked with a nearly 5 -fold higher risk of severe SARS-CoV-2 infection [14]. In our study, there were more severe patients in the returned-positive group than in the control group $(92.2 \%$ vs. $81.9 \%)$. This may partially explain why PCT was higher in returned-positive group.

Inflammatory markers have been considered as important predictors for the severity of the COVID-19. Elevation of serum levels of pro-inflammatory cytokines such as IL- 6 have been observed in severe COVID-19 patients [8, 15], which is commonly accompanied by higher CRP [16]. In our study, however, no consistent higher levels were found for CRP, IL-4 and IL-6 in the returned-positive group with more severe patients. Similarly, the CD3 + and CD8 + counts only showed significant differences in trends. Given the small number of patients doing these laboratory examinations in our study, large-scale studies are warranted to verify these associations. 
Two consecutively negative results of the nucleic acid test with a 24-hour interval are considered as clearance of COVID-19 [6]. Returned-positive or reoccurrence phenomena of SARS-CoV-2 among COVID19 patients have been reported in several counties, which has been considered as a failure of testing in a recent study [17]. The testing results of that previous study only indicate the presence of the SARS-CoV-2 virus in patients with moderate symptom, without quantitative evidence on any immunologic biomarkers. It is thus too simplistic to blame the returned-positive phenomenon among COVID-19 patients during the hospitalization to the false-negative testing or failure sampling. Our study, for the first time, provides quantitative evidence from a large patient cohort and demonstrates that patients suffered from positive return during hospitalization are likely to have distinct immunoreactive intensity, as reflected by an increasing trend of anti-SARS-CoV-2 IgG and higher levels of anti-SARS-CoV-2 IgM. Considering the consistent results of anti-SARS-CoV-2 IgM, this biomarker may be more indicative for the potential of returned-positive.

Our study has several limitations. First, study participants were from a single hospital and were restricted to patients receiving at least three SARS-CoV-2 laboratory tests. Selection bias or survivor bias may thus

exist, although we found no statistical differences in baseline characteristics between those included and excluded. Secondly, the number of patients declined at around 45 days after infection, and this compromises the study power to obtain significant findings for certain biomarkers. Thirdly, data on clinical symptoms were not available in our study. Finally, the discharged patients were unable to followup, and more studies are needed for these patients after discharge.

\section{Conclusions}

Our study suggests that returned-positive patients have distinctive inflammation and immunologic markers during hospitalization. Returned-positive patients of SARS-CoV-2 during hospitalization are less likely to be explained by false-negative RT-PCR test, as C3, PCT, serum IgM and anti-SARS-CoV-2 IgM are all significantly higher in the returned-positive group. More RT-PCR tests are needed for COVID-19 patients with these biomarkers before discharge.

\section{Abbreviations}

SARS-CoV-2: severe acute respiratory syndrome coronavirus 2

COVID-19: coronavirus disease which is first reported in 2019

WHO: World Health Organization

RT-PCR: real-time reverse transcriptase polymerase chain reaction

IQR: interquartile range

SD: standard deviation 
C3: the third component of complement

CRP: C-reactive protein

PCT: procalcitonin

IL-4: interleukin-4

IL-6: interleukin-6

LYM: the counts of lymphocyte

WBC: white blood cell

Ig: immunoglobulin

RTP: returned-positive

AGPK: glutamic-oxalacetic transaminase

DBIL: direct bilirubin

GGT: galactosylhydroxylysyl glucosyltransferase

GLB: globin

TBIL: total bilirubin

CK: creatine phosphokinase

CKMB: myocardial enzyme

IP: serum inorganic phosphorus

UA: uric acid

Urea: blood urea

LAP: leucine aminopeptidase

$\mathrm{Hb}$ : hemoglobin

PLT: platelet count

MYO: myohemoglobin

CD4CD8+: CD4+ counts / CD8+ counts 
IFN_Y: interferon-Y

TNF_a: tumor necrosis factor-a

IL_2: interleukin-2

ultra_Tnl: high sensitive troponin

\section{Declarations}

\section{Ethics approval and consent to participate}

This study was approved by the clinical ethics committee of the Renmin Hospital of Wuhan University. Informed consent from individual patients about the data analysis was exempted. The anonymous analysis was performed among all patients.

\section{Consent for publication}

Not applicable.

\section{Availability of data and materials}

The datasets used and/or analyzed during the current study are available from the corresponding author on reasonable request.

\section{Competing interests}

All the authors declare that there is no conflict of interests.

\section{Acknowledgements}

Not applicable.

\section{Funding}

This work was funded by the National Natural Science Foundation of China (No: 81970331,81370337). The funding bodies played no role in the design of the study and collection, analysis, and interpretation of data and in writing the manuscript.

\section{Contribution}

$Y W, X C$ and $Y Z$ were joint co-first authors. WY and $X Z$ were corresponding authors, conceptualized, designed the study, and made final approve of the manuscript before submission. YW, XC, YZ and HC conducted the statistical analysis and data interpretation and drafted the manuscript. $\mathrm{HY}$ and $\mathrm{XW}$ made clinical approve. All authors contributed interpretation of results. QZ, DL, ZN, HY, XW, HZ, TL and BZ collected data. 


\section{References}

1. WHO: Coronavirus disease (COVID-19) Situation Report - 136. In.; 2020.

https://www.who.int/emergencies/diseases/novel-coronavirus-2019/situation-reports/;(2020). Accessed 5 August, 2020.

2. McCloskey B, Heymann DL: SARS to novel coronavirus - old lessons and new lessons. EPIDEMIOL INFECT 2020, 148:e22.

3. Du Z, Wang L, Cauchemez S, Xu X, Wang X, Cowling BJ, Meyers LA: Risk for Transportation of Coronavirus Disease from Wuhan to Other Cities in China. EMERG INFECT DIS 2020, 26(5):10491052.

4. China. NHCO: Interim guidance for novel coronavirus pneumonia (Trial Implementation of Sixth Edition). http://www.gov.cn/zhengce/zhengceku/2020-02/19/content_5480948.htm; (2020). Accessed 3 May, 2020.

5. Xie X, Zhong Z, Zhao W, Zheng C, Wang F, Liu J: Chest CT for Typical 2019-nCoV Pneumonia: Relationship to Negative RT-PCR Testing. RADIOLOGY 2020:200343.

6. Lan L, Xu D, Ye G, Xia C, Wang S, Li Y, Xu H: Positive RT-PCR Test Results in Patients Recovered From COVID-19. JAMA 2020.

7. Organization. WH: Clinical management of severe acute respiratory infection when novel coronavirus (2019-nCoV) infection is suspected: interim guidance, 28 January 2020[R].

8. Huang C, Wang Y, Li X, Ren L, Zhao J, Hu Y, Zhang L, Fan G, Xu J, Gu X et al: Clinical features of patients infected with 2019 novel coronavirus in Wuhan, China. LANCET 2020, 395(10223):497-506.

9. Duan K, Liu B, Li C, Zhang H, Yu T, Qu J, Zhou M, Chen L, Meng S, Hu Y et al: Effectiveness of convalescent plasma therapy in severe COVID-19 patients. Proc Natl Acad Sci U S A 2020, 117(17):9490-9496.

10. Zeng QL, Yu ZJ, Gou JJ, Li GM, Ma SH, Zhang GF, Xu JH, Lin WB, Cui GL, Zhang MM et al: Effect of Convalescent Plasma Therapy on Viral Shedding and Survival in Patients With Coronavirus Disease 2019. J INFECT DIS 2020, 222(1):38-43.

11. Shen C, Wang Z, Zhao F, Yang Y, Li J, Yuan J, Wang F, Li D, Yang M, Xing L et al: Treatment of 5 Critically III Patients With COVID-19 With Convalescent Plasma. JAMA 2020.

12. Long QX, Tang XJ, Shi QL, Li Q, Deng HJ, Yuan J, Hu JL, Xu W, Zhang Y, Lv FJ et al: Clinical and immunological assessment of asymptomatic SARS-CoV-2 infections. NAT MED 2020.

13. Gralinski LE, Sheahan TP, Morrison TE, Menachery VD, Jensen K, Leist SR, Whitmore A, Heise MT, Baric RS: Complement Activation Contributes to Severe Acute Respiratory Syndrome Coronavirus Pathogenesis. MBIO 2018, 9(5):e1718-e1753.

14. Lippi G, Plebani M: Procalcitonin in patients with severe coronavirus disease 2019 (COVID-19): A meta-analysis. CLIN CHIM ACTA 2020, 505:190-191.

15. Xu Z, Shi L, Wang Y, Zhang J, Huang L, Zhang C, Liu S, Zhao P, Liu H, Zhu L et al: Pathological findings of COVID-19 associated with acute respiratory distress syndrome. Lancet Respir Med 2020, 
8(4):420-422.

16. Cao X: COVID-19: immunopathology and its implications for therapy. Nature reviews. Immunology 2020, 20(5):269-270.

17. Xiao AT, Tong YX, Zhang S: False-negative of RT-PCR and prolonged nucleic acid conversion in COVID-19: Rather than recurrence. J MED VIROL 2020.

\section{Figures}

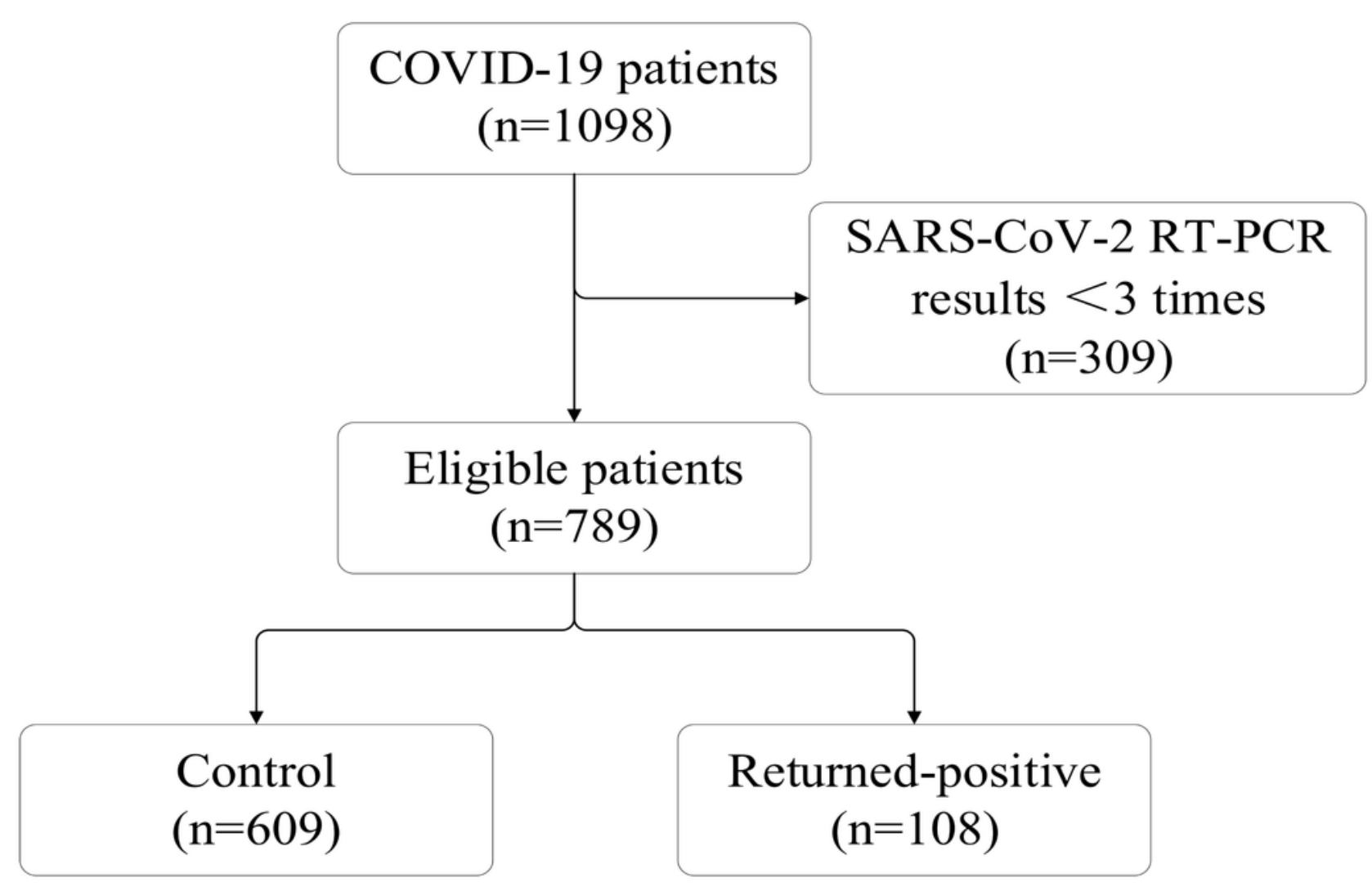

Figure 1

Flow chart of enrollment. 

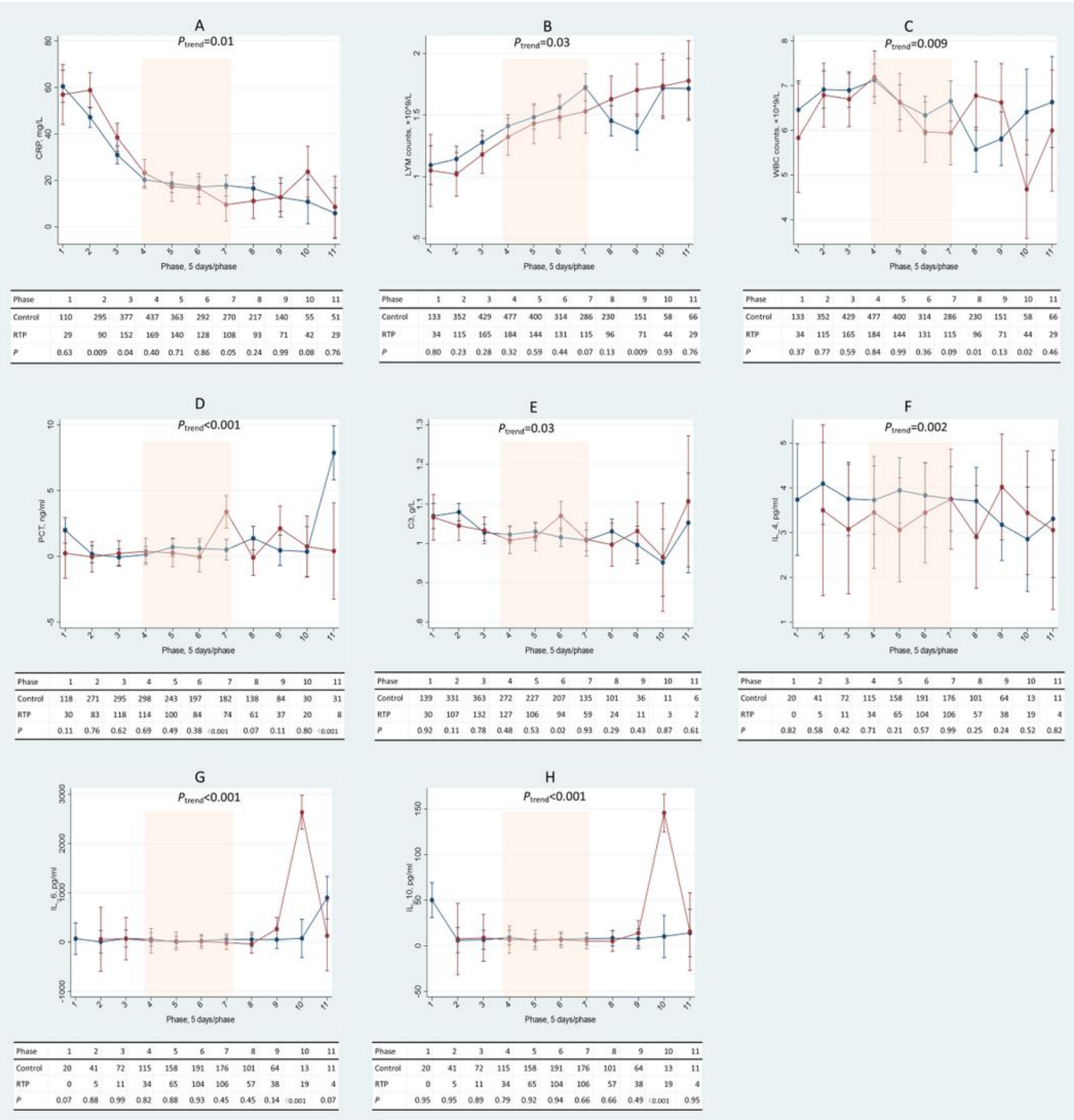

$\longrightarrow$ RTP (Returned-positive) $\longrightarrow$ Control $\quad \longrightarrow$ Returned-positive Period

\section{Figure 2}

The lab-inflammatory markers with significant different trends during hospitalization between two groups of COVID-19 patients Returned-positive period stands for the IQR of the time of COVID-19 patients appeared with returned-positive phenomenon. Returned-positive was defined as patients with two consecutive negative RT-PCR results of SARS-CoV-2 tests followed by a positive result, with an interval of over 24 hours between RT-PCR tests. The rest patients were defined as the control group. From the date of 
confirmed diagnosis of SARS-CoV-2 infection, the course of disease (days) was ordinally categorized into phases by an interval of 5 days.
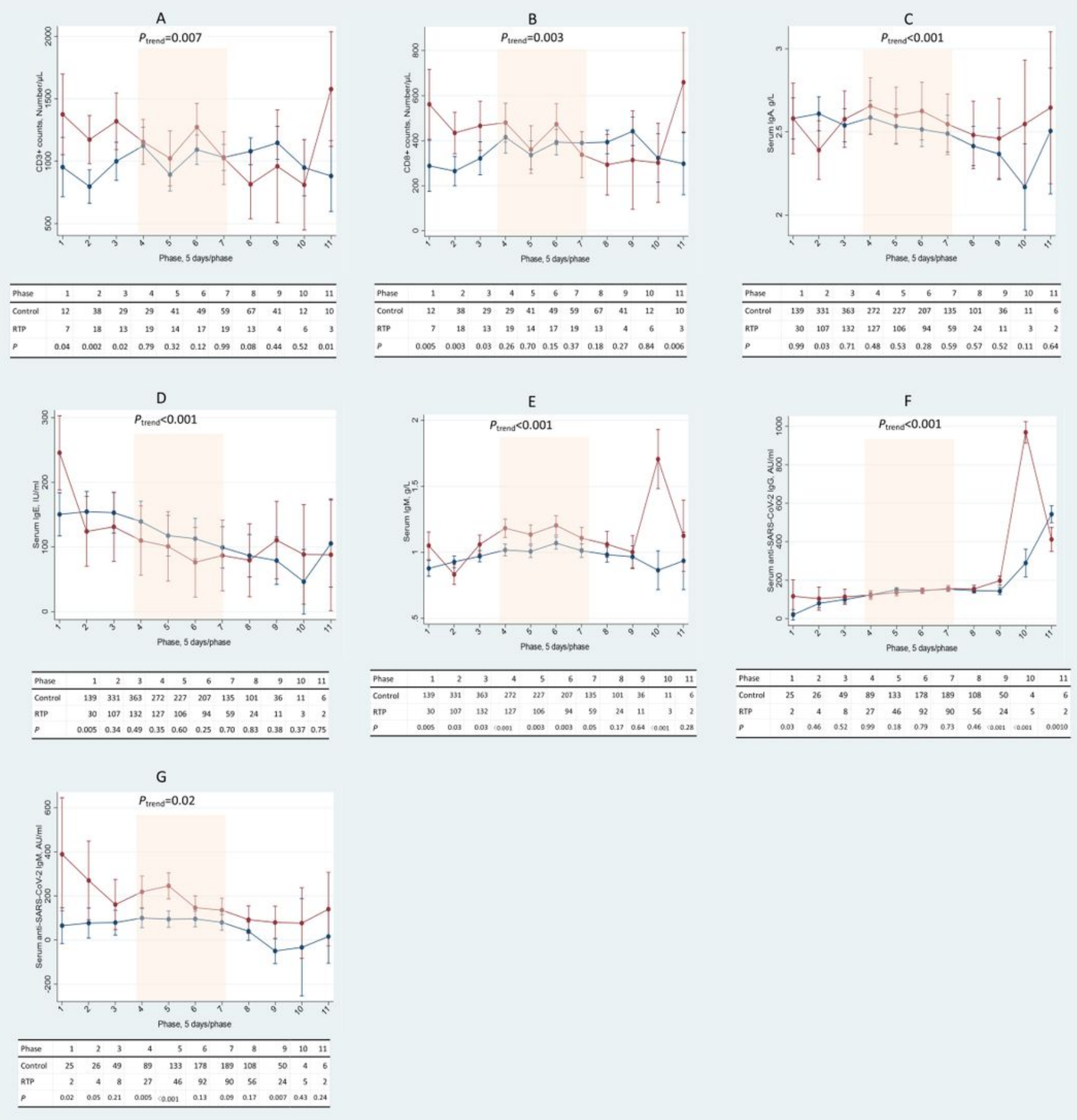

$\longrightarrow$ RTP (Returned-positive) $\longrightarrow$ Control $\quad \longrightarrow$ Returned-positive Period

\section{Figure 3}

The lab-immunologic markers with significant different trends during hospitalization between two groups of COVID-19 patients Returned-positive period stands for the IQR of the time of COVID-19 patients appeared with returned-positive phenomenon. Returned-positive was defined as patients with two 
consecutive negative RT-PCR results of SARS-CoV-2 tests followed by a positive result, with an interval of over 24 hours between RT-PCR tests. The rest patients were defined as the control group. From the date of confirmed diagnosis of SARS-CoV-2 infection, the course of disease (days) was ordinally categorized into phases by an interval of 5 days.

\section{Supplementary Files}

This is a list of supplementary files associated with this preprint. Click to download.

- AdditionalFile1.docx 